\title{
On the Treatment of Trachoma.
}

Professor SatTler, of Prague, has recently published a shortmonograph, entitled Die Trachombehandlung einst und jetzt (Fischer, Berlin, 1891). In this work, before proceeding to give an account of the treatment which he adopts, and the results obtained by it, he gives a most admirable résumé of the almost innumerable means which have formerly been used. Amongst the Egyptians, 1500 years before Christ, solutions of acetate of copper seem to have constituted the chief local application. The treatment adopted by Hippocrates, and which seems to have been practised for centuries, consisted in removing the granulations, and then applying the actual cautery or caustics to the raw surface.

During the period which intervened between the decay of ancient medicine and the beginning of the present century the references made in medical literature to trachoma are so ineagre and indefinite, that it has been held by. many authorities that the disease was first introduced into Europe on the return of the English, French, and Italian troops in 1801. Sattler gives good reasons for believing that this is a mistake, and that, notwithstanding the great epidemics which originated from this cause, and which attracted the attention, not only of military and civil surgeons, but of the different European governments, the disease had for a long time existed in Europe. Further, he points out that much of the so-called Egyptian or military ophthalmia included cases of severe catarrhal and gonorrhoeal conjunctivitis, the conncction of which with gonorrhœa was but imperfectly understood.

The disease, as it occurred in the epidemics thus introduced into Europe, was very generally treated by means of copious bloodletting, either by arteriotomy or phlebotomy. The patients were bled to from thirty to fifty or even sixty ounces. In addition, they were frequently subjected to large blisters between the shoulders, etc. Only a few recognized the importance of ventilation and open-air treatment. When the acute symptoms had subsided a whole host of topical agents were in use: acetate of lead, sulphate of zinc, weak solutions of copper salts and alum, weak acids, laudanum, mercucurial preparations, etc. Subsequent vegetations were almost invariably treated by removal. In England, especially, the granulations seem to have been, at that time, always excised. In other countries the same effect was often produced by the use of strong caustics. Gradually, and not till after 1830, there came a reaction against the excessive and often repeated bleeding.

In 1835 , Kerst, a Dutch military surgeon, introduced the so. called abortive treatment by cauterization of the conjunctiva with lapis divinus. Although this treatment; as adopted by Kerst, was not strictly followed by many of his contemporaries, it seems to have led to a more rational use of nitrate of silver, which soon became, as, indeed, it still remains, one of the chief topical agents employed. The value of sulphate of copper and acetate of lead seem only to have been recognized some ten years later.

These three substances have, for the last thirty or forty years, very properly, Sattler considers, been held in the greatest favor as local applications on trachoma in all civilized countries. He considers it certain that, by their 
use, many cases of trachoma have been cured-i.e., have been brought to a favorable termination, and recurrences permanently checked. In his opinion, therefore, those who, like Jacobson, hold that nothing but operative treatment is of any avail, go decidedly too far. Their main disadvantage is the length of time during which the treatment has to be carried on. This, in itself, as it demands great patience both in the surgeon and the patient, must necessarily frequently be the cause of failure. The frequent disappearance of patients before a cure is effected, renders it difficult, or impossible, to root the disease out of infected localities. Sattler very properly lays stress on the uselessness of giving trachoma patients ointments and drops with which to treat themselves.

These considerations have given rise in many to the desire to shorten the treatment in some way or other. The conviction, which has more and more gained ground, that the vesicular or glandular, or so-called trachoma granulations, constitute the specific element of the disease, has led to different methods being adopted to remove or destroy these follicles.

The operative treatment of trachoma most in vogue at the present time is the excision of more or less of the affected conjunctiva, and the squeezing or raking out of the follicles.

The advantages which have been claimed for the different methods of excision of conjunctiva are the following: (1) Considerable shortening of the treatment, so that in uncomplicated cases patients are able to resume work three or four weeks afterward; (2) The subsequent disappearance of the follicles remaining in the conjunctiva of the lid; (3) A favorable and speedy effect on the corneal complications; (4) The rarity of the manifestation of recurrence; (5) The absence of subsequent changes in the position of the lids, or of cicatrices which cause any subjective disturbances, or interfere with the movements of the eyes or lids.

Sattler is able, from a large experience of the treatment by excising the conjunctival fold to confirm, in a general way, the justice of the above claims. He remarks that when, however, in order to avoid making a too complete excision, and trusting to its favorable influence on the remaining follicles, one allows groups of follicles to remain in the lower lid, conjunctiva bulbi, or caruncle, and discharges the patients before they have been caused to disappear completely by proper topical treatment, severe recurrences are by no means excluded. He considers that the chief objection which has been raised against the excision treatment, viz., that the cicatricial contractions thus caused might be worse than those resulting from tbe trachoma process itself, is not founded on fact, but only entertained by those who have had no experience of it. An excision of a fold two to five millimetres in breadth produces absolutely no defect, and much more extensive excisions, undertaken in the more advanced cases of trachoma, do not.cause the supposed interference with the normal movements, provided they be made superficially, and do not involve the tendon of the levator, or its insertion.

Whilst, however, in Sattler's opinion, the excision method of treatment is an undoubted advance, he considers that the question may well arise whether a rapid radical cure of trachoma may not be effected by means which are more consistent with the fundamental principles of surgery. In performing excision, we remove not only the specific new formation of the trachomatous 
process, but, in addition, other parts which, though perhaps they may not be in a normal state, are yet only secondarily affected in a manner from which they are quite capable of recovering. He considers that the pathology of trachoma affords a well-grounded indication for the early and complete removal of the follicular deposits, while at the same time avoiding as much as possible any interference with the other tissues. The history of such attempts, and the different ways in which they have been made, is a long one, and cannot well be condensed within the limits of an abstract.

The method employed by Sattler consists in rupturing the follicles with a eataract needle, and scooping out their contents with a fine, specially constructed sharp spoon. This instrument is circular, and has a diameter of from two to four millimetres. In shelling out the contents of the follicles in the conjunctival folds, the tissues have to be put on the stretch by means of a fixation forceps. Sattler lays great stress upon eradicating all the follicles at one sitting, using a general anæsthetic when necessary. The reaction is usually inconsiderable, and the subsequent treatment consists in washing out the conjunctival sac with corrosive sublimate lotion.

\section{DISEASES OF THE LARYNX AND CONTICUOUS STRUOTURES.}

UNDER THE CHARGE OF

J. SOLIS-COHEN, M.D., OY PEILADRL.PHIS.

\section{I laryngeal Paralyses.}

An instance of bilateral paralysis of the recurrent laryngeal nerves in a tuberculous subject, attributed, in the absence of all indications of aortic aneurism, to compression by tuberculous mediastinal glands while the paralysis remained unilateral, and to a tumor in the posterior mediastinum when it became bilateral, and proving on the autopsy to have been due to aneurism of the aorta after all, has been reported by Dr. EUGENE FRÄANKEL, of Hamburg (Deutsche med. Wochensehrift, 1890). The aneurism, which was located between the left carotid and subclavian arteries, extended as far as the isthmus of the thyroid gland, which had undergone atrophy to a thin remnant by the pressure. The left recurrent nerve was firmly united to the aneurismal sac, and was transformed, about two inches above its point of recurrence, into a thin, wholly atrophied thread. So, likewise, with the right recurrent and pneumogastric nerves. A firm thrombus was discovered in the innominate vein at the junction of the jugular vein.

Similar instances of aortic aneurism without the ordinary physical signs of aneurism are not uncommon. Paralysis of the recurrent laryngeal nerves, in the absence of positive indications of different origin, is usually presumptively due to pressure from an aneurism. 